\title{
Highly Sensitive Imaging of Cancer with Functional Nanoparticles
}

\author{
Kohsuke Gonda ${ }^{1,2, *}$, Yoh Hamada ${ }^{2}$, Narufumi Kitamura ${ }^{2,3}$, Hiroshi Tada ${ }^{3}$, Minoru Miyashita ${ }^{3}$, \\ Takashi Kamei $^{4}$, Takanori Ishida ${ }^{3}$, and Noriaki Ohuchi ${ }^{2,3}$ \\ ${ }^{1}$ Department of Medical Physics, Graduate School of Medicine, Tohoku University, \\ ${ }^{2}$ Department of Nano-Medical Science, Graduate School of Medicine, Tohoku University, \\ ${ }^{3}$ Department of Surgical Oncology, Graduate School of Medicine, Tohoku University, \\ ${ }^{4}$ Department of Advanced Surgical Science and Technology, Graduate School of Medicine, Tohoku \\ University, Seiryo-machi, Aoba-ku, Sendai 980-8575, Japan \\ *gonda@med.tohoku.ac.jp
}

\begin{abstract}
It is important for cancer therapy to understand cancer mechanisms and develop a diagnostic method. We have developed a method for in vivo imaging with very high spatial accuracy $(\sim 9 \mathrm{~nm})$ under a confocal microscope and succeeded in tracking the membrane protein during metastasis in living mice. We found that the tumor cells showed increases in membrane fluidity (over 1000-fold) and formed local pseudopodia in the process of metastasis, suggesting that membrane fluidity and morphological changes are critical for metastasis. To develop a novel immunohistochemistry (IHC), we newly-made organic fluorescent material-assembled nanoparticles. These nanoparticles have 1000-fold greater fluorescent intensity compared to representative organic fluorescent material. Consequently, the fluorescence of these nanoparticles exhibited a significantly high signal-to-noise ratio on IHC-imaged cancer tissue, including high-level autofluorescence. The IHC method using these nanoparticles was applied for the identification of estrogen receptor-expression levels in breast cancer tissue. The results demonstrated that the diagnostic accuracy and quantitative sensitivity were greatly improved compared to previous IHC methods. This technique would be useful for the prediction of clinical responses to ER-targeted therapy.
\end{abstract}

Keyword: nanoparticle, imaging, cancer, metastasis, immunohistochemistry

\section{Introduction}

In cancer mechanisms, metastasis is one of the most pathological properties of malignant cancer cells. During metastasis, membrane dynamics are significantly altered in metastatic cancer cells [1]. Many studies using cultured cells have suggested that metastatic cancer cells form pseudopodia termed filopodia, lamellipodia and invadopodia [2]. Additionally, greater membrane fluidity is thought to enhance the malignancy of cultured cancer cells [3]. High membrane fluidity is coupled to increased diffusion speed of membrane proteins. Greater diffusion speed accelerates the reaction rate between adhesion proteins and their extracellular substratums. Thus, to elucidate the mechanisms of cancer metastasis, analysis of membrane protein dynamics during metastasis is crucial. In living tumors in vivo, there are blood vessels and three-dimensional communication systems between cells, unlike in in vitro cultures. It is therefore essential that in vivo membrane morphology and fluidity based on membrane protein dynamics is clarified. 
Previous studies used imaging of GFP- or luciferase-expressing cancer cells in vivo to examine the behavior of metastatic cancer cells. However, as the spatial precision of such imaging is limited to the micrometer level and single-molecule imaging is impossible, the details of in vivo dynamics of individual membrane proteins remain unknown. Here, we have developed a method to image a tumor cell membrane protein with antibody-conjugated quantum dots (QDs). We used this technique to visualize the details of membrane fluidity and morphology during metastasis in living mice with a spatial precision of 7-9 nm [4].

To develop diagnostic methods of cancer tissues, we improved immnnohistochemistry using biomarkers. The estrogen receptor (ER), progesterone receptor (PgR) and human epidermal growth factor 2 (HER2) were the first biomarkers for breast cancer to be recommended for routine clinical use. ER is a member of the nuclear hormone family of intracellular receptors, and it plays a critical role in cancer growth. Approximately $70 \%$ of breast cancer cases are ER-positive [5].

Pathological examination is the gold standard for cancer diagnosis. IHC with 3,3'-diaminobenzidine (DAB) (IHC-DAB) is the most conventional IHC protocol. However, the intensity of DAB staining depends on the enzyme activity of horseradish peroxidase (HRP). Therefore, the staining intensity is significantly influenced by reaction time, temperature and HRP substrate concentrations. The intensity of DAB staining often differs between experimenters. IHC using fluorescent molecules or particles as labels is an effective quantitative use of IHC because the intensity of fluorescent materials is proportional to the intensity of photon excitation energy, which is an irreversible chemical reaction. However, high levels of tissue autofluorescence have impeded the development of this technique. Therefore, the creation of a novel bright fluorescent material suitable for IHC was required to improve fluorescent labeling IHC for the quantitative diagnosis of ER expression. We here prepared organic fluorescent material-assembled nanoparticles for IHC. The fluorescent intensity of the novel nanoparticles was approximately 1000 -fold greater than that of general organic fluorescent molecules, and the level of ER expression was quantified with much greater accuracy compared to IHC-DAB [6].

\section{Materials and Methods}

2.1. Optical System with Confocal Microscope

The optical system for observation of the fluorescence of QDs consisted primarily of an epi-fluorescent microscope (IX-71, Olympus) with modifications, a Nipkow disk-type confocal unit (Yokogawa), and an electron multiplier type charge-coupled device camera (EM-CCD, Andor Technology). For in vivo imaging, in order to remove the oscillation of heartbeat and respiration in observations, an aluminum stage was developed and attached to the above microscopy system.

\subsection{In vivo Imaging}

PAR1-expressing KPL (PAR1-KPL) cells were transplanted subcutaneously into the skin of female SCID mice at 5-7 weeks of age. Five to ten weeks after transplantation, anti-PAR1 antibody-conjugated QDs (anti-PAR1 QDs) were injected into the tail vein of the mice. The mice labeled with the probe were placed under anesthesia, and the anesthetized condition was maintained for the course of the imaging session. Minimal surgery was performed to expose the living tumor by removing the skin with as little damage to the surrounding blood vessels as possible. The position of QDs on the tumor cell membrane was tracked using a previously described single-molecule tracking method [7]. Animals were used in accordance with guidelines approved by the committee on animal experiments of Tohoku University.

2.3. Preparation of Organic Fluorescent Material-Assembled Nanoparticles

Tetramethylrhodamine (TMR) was mixed with 3-aminopropyltriethoxysilane in dimethylformamide. This mixture was incubated with a buffer containing ethanol, tetraethylorthosilicate, ultrapure deionized water, and ammonia water. The incubated sample was centrifuged. The precipitates were washed with ethanol and water using centrifugation. TMR-assembled nanoparticles were obtained using this process. To coat the surface of TMR-assembled nanoparticles with streptavidins via polyethylene glycol (PEG) chains, TMR-assembled nanoparticles were treated with PEG chains, in PBS containing EDTA. The PEG 
chain-coated TMR-assembled nanoparticles were centrifuged. The sample was incubated with streptavidins reduced by dithiothreitol. The sample was washed and redispersed in PBS containing EDTA. Streptavidin-coated TMR-assembled nanoparticles (Avi-TMR particles) were obtained for immunostaining.

\subsection{Immunohistochemistry}

Breast cancer tissue specimens were purchased. The specimens were deparaffinized in xylene and hydrated in graded alcohols and distilled water. Endogenous horseradish peroxidase (HRP) activity was blocked. The antigen retrieval was performed. The samples were incubated with BSA and immunostained using a rabbit anti-ER primary antibody. Then the samples were incubated with a biotinylated goat anti-rabbit IgG secondary antibody. The samples were incubated with streptavidin-conjugated HRP or Avi-TMR particles. Samples that were incubated with streptavidin-conjugated HRP were treated with a DAB chromogen reagent. In addition to the staining of biomarkers, the investigation of nuclear morphology using counterstaining with hematoxylin is important in cancer diagnosis using IHC. All samples were stained with hematoxylin after DAB and Avi-TMR particle staining.

\section{Results}

3.1. In vitro and in vivo Nano-Imaging of Membrane Fluidity of Cancer Cells during Metastasis with Fluorescence Nanoparticles

The membrane fluidity in PAR1-KPL cells were investigated in vitro by imaging PAR1 movement labeled with anti-PAR1-QDs. To detect the movement of the anti-PAR1-QDs on membrane, QDs was tracked referencing the membrane position observed by phase contrast images. The movement of anti-PAR1-QDs was observed on an immobile membrane region and mobile lamellipodia. The center position of fluorescence intensities of anti-PAR1-QDs was calculated by fitting the intensity profiles to two dimensional Gaussian curves [7]. The center was moved randomly along the membrane. To quantitatively analyze the diffusional movements of anti-PAR1-QDs, a mean square displacement (MSD) of the QD position was analyzed. The diffusion constant for QDs on the immobile regions of PAR1-KPL cells were $2.2 \times 10^{4} \mathrm{~nm}^{2} / \mathrm{s}$. In contrast to this, the diffusion constant of QDs on mobile lamellipodia of PAR1-KPL cell $\left(1.2 \times 10^{5}\right.$ $\mathrm{nm}^{2} / \mathrm{s}$ ) was 5 fold faster than that of immobile cell edges (Fig. 1A). The faster diffusion velocity of PAR1 on mobile edge suggests that the membrane fluidity increases on the locally pseudopodial formation.

Next, the anti-PAR1-QDs were injected into the tail vein of tumor-bearing mice. After injection, QDs bound to the tumor stroma were imaged under a optical system. The center of the QD image was calculated. We measured values of about $7 \mathrm{~nm}$ for the $\mathrm{X}$-axis and about $9 \mathrm{~nm}$ for the Y-axis, indicating that we can track the movement of PAR1 labeled with anti-PAR1-QDs with a spatial precision of 7-9 $\mathrm{nm}$ in vivo.

We observed the in vivo membrane dynamics of metastatic cancer cells in four regions: far from the blood vessel in tumors, near the vessel, in the bloodstream, and adherent to the inner vascular surface in normal tissues near tumors. These locations represent the process of cancer metastasis (Fig. 1B-F). To analyze the diffusional movement of anti-PAR1-QDs quantitatively, MSD of QD position was analyzed. The diffusion constant of PAR1 on cells far from tumor vessels was low, at $72 \mathrm{~nm}^{2} / \mathrm{s}$ (Fig. 1B). In cells near blood vessels, the diffusion constant of PAR1 facing the vessel $(2.2 \times$ $10^{4} \mathrm{~nm}^{2} / \mathrm{s}$ ) (Fig. 3C), was 28-fold greater than that in the tail of the same cell $\left(8.0 \times 10^{2} \mathrm{~nm}^{2} / \mathrm{s}\right)$ (Fig. $3 C$ ) and 300-fold greater than that of cells far from the vessel $\left(72 \mathrm{~nm}^{2} / \mathrm{s}\right)$. These results suggest that the diffusion of PAR1 on vessel-facing membranes of cells near blood vessels might be accelerated by an attractant released from the vessel. Next, we visualized cancer cells in vessels, cells in the bloodstream within tumors and cells adhering to the inner vascular surface in normal tissues near tumors. In vessels, the QDs on the cell membrane in the bloodstream moved along the cell edge and the diffusion constant was $8.2 \times 10^{4} \mathrm{~nm}^{2} / \mathrm{s}$, which was 1100 -fold greater than that observed for cells far from a tumor vessel (Fig. 1D). In the cells adhering to the inner vascular surface without directional movement, the QDs moved along the cell edge and the diffusion constant of PAR1 was $3.9 \times 10^{4} \mathrm{~nm}^{2} / \mathrm{s}$; this was two-fold lower than in the bloodstream (Fig. 1E). Moreover, in the cells on the inner vascular surface showing directional migration, the diffusion constant of PAR1 $(3.6 \times$ $10^{3} \mathrm{~nm}^{2} / \mathrm{s}$ ) was 23-fold less than that in cells in the bloodstream (Fig. 1F). In addition, the PAR1 diffusion constant on the lamellipodia $\left(8.2 \times 10^{3}\right.$ $\mathrm{nm}^{2} / \mathrm{s}$ ) was two-fold greater than that of other 
regions in the migrating cells.

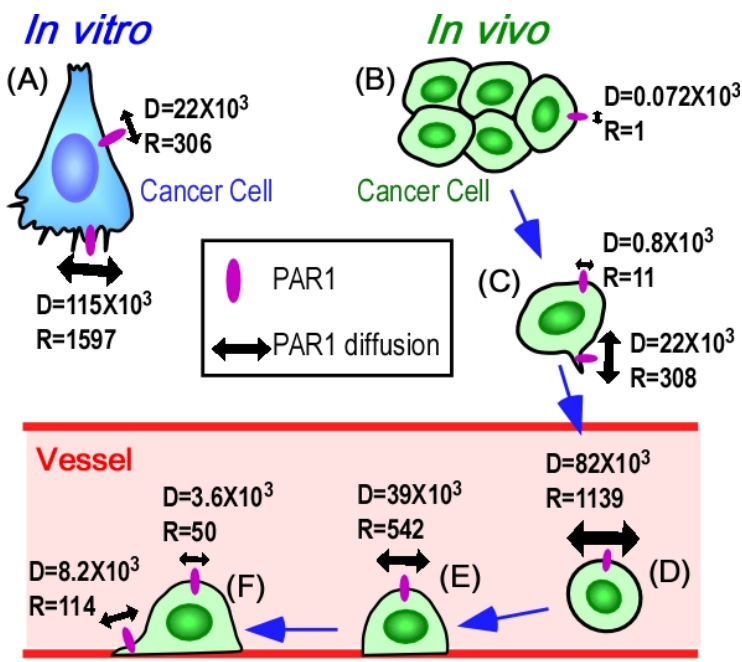

Fig. 1. A model for membrane dynamics in metastatic cancer cells. (A) Cancer cells in vitro. (B-F) Cells in vivo during metastasis. (B) Cells far from vessels. (C) Cell near vessels. D, Cell in the bloodstream. (E) Cells adhering to the inner vascular surface without directional migration. (F) Cells migrating directionally on the surface. Numerical values of " $D$ " show the diffusion coefficient $\left(\mathrm{nm}^{2} / \mathrm{s}\right)$ of PAR1 labeled with anti-PAR1-QDs. Numerical values of "D" reveal relative values of " $\mathrm{R}$ " when diffusion coefficient in cells far from vessels $\left(0.072 \times 10^{3} \mathrm{~nm}^{2} / \mathrm{s}\right)$ was defined as 1 .

3.2. Nano-imaging of Human Cancer Tissues to Diagnose Expression Level of Cancer-related Protein with Fluorescence Nanoparticles

To develop IHC with fluorescence, the creation of a novel bright fluorescent material suitable to IHC was required. We selected an organic fluorescent molecule, TMR, and we prepared Avi-TMR particles. The Avi-TMR particle consisted of hundreds of thousands of TMRs, and its surface was coated with streptavidins via PEG chains (Fig. 2A). The SEM image revealed that the TMR particles were quite uniform in size, and the average size was $115 \mathrm{~nm}$ (variation coefficient, 12\%). The fluorescence spectral analysis demonstrated that the peak of $532 \mathrm{~nm}$ light-excited spectral patterns of the Avi-TMR was at approximately $580 \mathrm{~nm}$. The fluorescent intensity of Avi-TMR particles was about 1000-fold greater than that of 532 nm-excited TMR. The data suggested that the Avi-TMR particles possessed a significantly higher fluorescence intensity than the autofluorescence intensity of tissues.

The detection of ER using IHC in breast cancer tissue is a strong predictive marker for the response to hormone-based therapies, such as tamoxifen [5]. However, IHC-DAB is a poor quantitative method. Therefore, the quantitative sensitivity of the existing diagnostic methods for ER detection is quite low. We immunostained breast cancer tissue specimens that expressed ERs at high or low levels with Avi-TMR particles. We observed differences in the quantitative sensitivity of DAB staining and fluorescent nanoparticle staining. ER is a member of the nuclear hormone family of intracellular receptors. Therefore, nuclear staining is observed in cancer cells that are positively immunostained with an anti-ER primary antibody. DAB- and Avi-TMR particle staining exhibited non-staining or weak staining intensity in low-level ER-expressing tissues, respectively and strong intensity in high-level ER-expressing tissues (Fig. 2B, C). These results suggested that both IHC methods specifically stained ER-expressing breast cancer tissues. The fluorescence intensity of Avi-TMR particles was about 1000-fold greater than that of TMR. Therefore, we obtained significantly higher signal-to-noise ratios for the Avi-TMR particles in the presence of autofluorescence.

Adjacent tissues that expressed high ER levels were immunostained with DAB or Avi-TMR particles to compare the quantitative sensitivity of the two methods, and the same regions of both tissues were observed (Fig. 2B, C). Previous studies did not consider the ER-expression levels of each cell. Therefore, it is difficult to diagnose ER levels with high accuracy and apply the pathological information to ER-targeted medical therapy. Figure 2 illustrates that DAB and Avi-TMR particles stained the same regions in adjacent tissues. Each image (Fig. 2B, C) was converted into a gray scale image, and the staining intensity per cell nucleus was measured. A wide range of variations in nuclear size was observed in tissue sections. Therefore, the staining intensities were calculated as gray values per pixel in the cell nucleus to avoid a staining intensity value that depended on cell nuclear size. Moreover, the value of each data set was multiplied by a constant coefficient, and the mean value of each data set was adjusted to 1000 as an arbitrary unit (a.u.) so that the DAB- and Avi-TMR particle staining intensity data could be compared on the same scale (Fig. 2D). ER expression levels vary widely according to cell heterogeneity in tissues. Therefore, a wide range in staining intensity values may be produced by the accuracy and quantitative 
sensitivity of IHC. The results demonstrated that the standard deviations of Avi-TMR particle staining images was much larger than the $\mathrm{DAB}$ staining images (Fig. 2D).

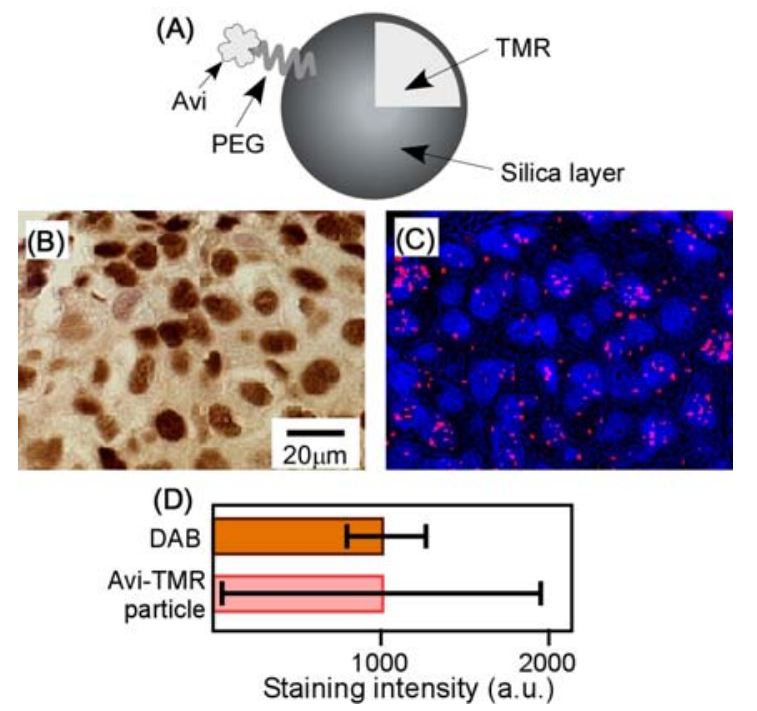

Fig. 2. Immunohistochemistry of ER with Avi-TMR nanoparticles. (A) Schematic for the Avi-TMR particle preparad. Adjacent tissues expressing ER at high level were immunostained. One was stained with DAB- and hematoxylin (B). Another was stained with Avi-TMR particles and hematoxylin (C). After that, a same region of both staining tissues were observed. DAB-staining image (A) was converted into their gray scale images and then the gray scale tone was inverted. Avi-TMR particles-staining image was were converted into their gray scale image. To measure the Avi-TMR particles' fluorescent signal in cellular nuclear, the nuclear region was surrounded with ROI that was determined by hematoxylin-staining image. The mean value of DAB- staining intensity (B) or Avi-TMR particles-staining intensity (C) were adjusted to 1000 as arbitrary unit (D). Error bars (C) indicate standard deviation that shows the wide range of variations in staining intensity value.

\section{Discussion}

4.1. Clarification of Membrane Fluidity of Cancer Cells in vivo during Metastasis

PAR1 diffusion at the mobile edge was greater than at the immobile edge in vitro and in vivo (Fig. $1 \mathrm{~A}, \mathrm{C}, \mathrm{F}$ ). As membrane protein movement is coupled with actin filament density underneath the cell membrane, membrane fluidity and actin dynamics might synergistically activate local pseudopodial formation. In mice, cancer cells in colonies far from tumor blood vessels did not show active membrane dynamics (Fig. 1B). This might have been due to low local concentrations of signaling factors derived from blood components. The cells near blood vessels are likely attracted to the vessels by signaling factors, resulting in the formation of membrane protrusions (Fig. 1C). The concentration gradient of such signaling factors through the extracellular matrix might also result in increased membrane fluidity and actin dynamics within individual cells on sides facing the blood vessel. In the bloodstream, the membrane fluidity of cells was 1100 -fold higher than that of cells far from vessels (Fig. 1D). This increased diffusion was likely caused by the existence of fewer actin filaments underneath the cell membrane due to a lack of adhesion between cells and the extracellular substratum. Additionally, greater diffusion of membrane protein in vessels might enhance cancer metastasis to another organ by accelerating the reaction rate between adhesion proteins and their extracellular substratums. After adhering to the inner vascular surface, diffusion decreased (Fig. 1E, F). The reformation of the actin cytoskeleton underneath the cell membrane likely slowed the diffusion of PAR1. The membrane fluidity of the lamellipodia-like structure was two-fold higher than that of other regions (Fig. $1 \mathrm{~F})$. Similar results were also obtained in cultured cells (Fig. 1A). The diffusion constant of QDs on mobile lamellipodia (1.2 $\times 10^{5} \mathrm{~nm}^{2} / \mathrm{s}$ ) in vitro was five-fold greater than that on immobile cell edges $\left(2.2 \times 10^{4}\right.$ $\mathrm{nm}^{2} / \mathrm{s}$ ).

In conclusion, the membrane fluidity of metastasizing tumor cells increases at intravasation, peaks in blood vessels, decreases at extravasation and is higher in locally formed pseudopodia. Such dramatic changes in membrane fluidity and morphology can enable cancer cells to metastasize.

\subsection{Development of Pathological Diagnosis} with Fluorescence Nanoparticles.

We developed a new IHC technique using novel TMR-assembled nanoparticles. These nanoparticles have 1000-fold greater fluorescent intensity compared to organic fluorescent material. Consequently, the fluorescence of these nanoparticles exhibited a significantly high signal-to-noise ratio on 
IHC-imaged cancer tissue, including high-level autofluorescence. The results demonstrated that the diagnostic accuracy and quantitative sensitivity were greatly improved compared to previous IHC methods.

In addition, the investigation of nuclear morphology by counterstaining with hematoxylin is very important. Hematoxylin staining did not effect Avi-TMR particle staining patterns, which demonstrated that a biomarker diagnosis using Avi-TMR particles and nuclear morphology diagnosis using hematoxylin can be performed in identical tissue specimens.

DAB staining in low-level ER-expressing tissues was difficult to identify (data not shown). Longer reaction times are required to clearly observe low expression levels of ER using DAB staining. However, positive signals from Avi-TMR particles in low-level ER-expressing tissues were clearly observed. These results demonstrated that Avi-TMR particle staining quantitatively analyzed a wider range of ER expression levels compared to DAB staining.

These results demonstrate that the Avi-TMR particle-staining method greatly increased the accuracy and quantitative sensitivity of IHC compared to IHC-DAB. Enhancement of the diagnostic accuracy and quantitative sensitivity for ER will improve the predictive response to therapies targeting ERs and PgRs that are induced by a downstream ER signal.

\section{Conclusion}

It is important for cancer therapy to understand cancer mechanisms and develop a diagnostic method. The in vivo imaging of metastatic cancer cells with anti-PAR1-QDs showed that the tumor cells changed the membrane fluidity over 1000 times according to the location of cancer cells during metastasis and then formed locally pseudopodia in the process of metastasis [4].

The IHC method using novel Avi-TMR nanoparticles demonstrated that the diagnostic accuracy and quantitative sensitivity were greatly improved compared to previous IHC methods. This technique would be useful for the prediction of clinical responses to ER-targeted therapy [6].

\section{Acknowledgements}

A portion of this work was supported by a Grant-in-Aid for Scientific Research in Innovative Areas "Nanomedicine Molecular Science" (23107009) from the Ministry of Education, Culture, Sports, Science, and Technology, Japan (K.G.) and a Grant-in-Aid for the Comprehensive Research and Development of an Early Stage Diagnosis Method and Instruments to Treat Cancer from the New Energy and Industrial Technology Development Organization, Japan (K.G. and N.O.). We would also like to acknowledge the support of the Biomedical Research Core at the Tohoku University Graduate School of Medicine and Konica Minolta, Inc.

\section{References}

1. E. Sahai, Nature Rev. Cancer, 7 (2007) 737-749.

2. T.D.Pollard and G. G. Borisy, Cell, 112 (2003) 453-465.

3. F. R. Maxfield and I. Tabas, Nature, 438 (2005) 612-621.

4. K. Gonda, T. M. Watanabe, N. Ohuchi, and H. Higuchi, J. Biol. Chem., 285 (2009) 2750-2757.

5. A. M. Gown, Modern Pathol., 21 (2008) S8-S15.

6. K. Gonda, M. Miyashita, M. Watanabe, Y. Takahashi, H. Goda, H. Okada, Y. Nakano, H. Tada, M. Amari, and N. Ohuchi, Biochem. Biophys. Res. Commun., 426 (2012) 409-414.

7. T. M. Watanabe and H. Higuchi, Biophys J.,92 (2007) 4109-4120. 